\title{
Stationskonzepte \\ Neuverteilung der Aufgaben- und Kompetenzprofile
}

Stationen sind zentrale leistungserbringende Einheiten mit direktem Patientenkontakt. Zudem gleichen Sie nach Umsatz und Größe oft mittelständischen Unternehmen. Einer der entscheidenden Faktoren für die Wirtschaftlichkeit einer medizinischen Abteilung ist die Etablierung ihrer Station als zentrale Verantwortungseinheit mit klaren Führungsstrukturen, Aufgabenprofilen und Verantwortungsbereichen. Dies gilt umso mehr, da die gewachsenen, stationären Organisationsformen den aktuellen Anforderungen, wie steigender Arbeitsverdichtung und Fachkräftemangel, nicht länger gerecht werden können.

Der Wandel hin zu modernen und gleichzeitig betriebswirtschaftlich erfolgreichen Strukturen bedarf eines besonnenen Change Managements, das eine gesunde Balance zwischen den Bedürfnissen von Patienten und Mitarbeitern, aber auch der Erfolgswirksamkeit der neuen Stationsabläufe herstellt. Wesentliches Erfolgskriterium dafür die ist Neuverteilung der Aufgaben- und Kompetenzprofile im Rahmen gestufter Personalkonzepte.

Inselorganisationen aufheben - neue Führungsstrukturen schaffen

Kaum ein anderes Unternehmen zeichnet sich so stark durch Inselorganisationen aus wie ein Krankenhaus. Es gibt festgeschriebene Abteilungsgrenzen, Grenzen zwischen den Berufsgruppen, die in sich z. T. stark hierarchisch strukturiert sind und nicht zuletzt die Bereichsgrenzen zwischen Ambulanzen, Stationen, OPund Diagnostikeinheiten sowie weiteren am Behandlungsprozess Beteiligten. Insbesondere Stationen sind geprägt von einer hohen Anzahl dieser Schnittstellen organisatorische Rahmenbedingungen, die die Entwicklung abgeschotteter Verantwortungsbereiche unterstützen. Die Einführung erfolgreicher Stationsstrukturen erfordert einen Paradigmenwech-

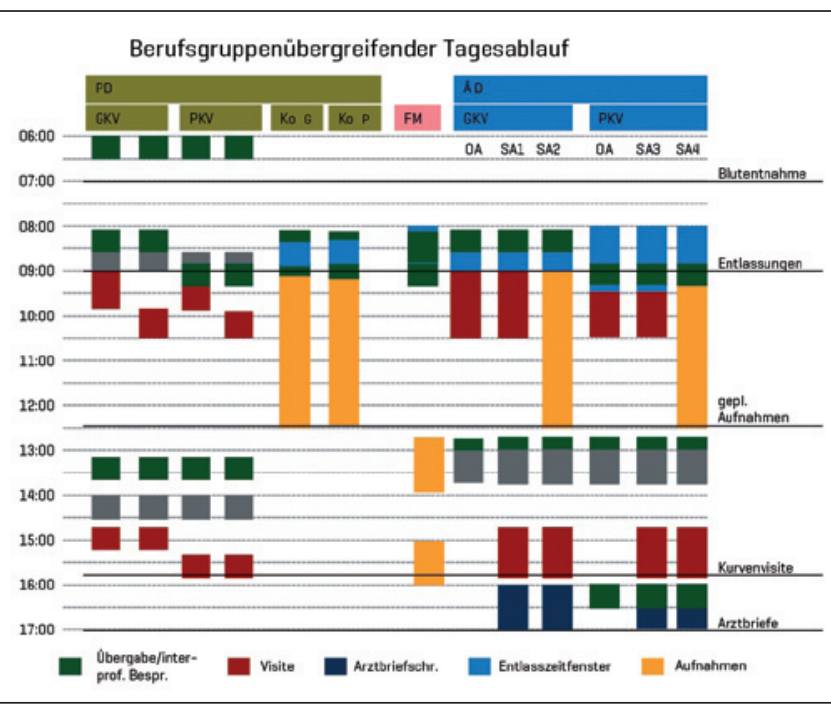

Abb. 1 Berufsgruppenübergreifender Tagesablauf sel, weg von der „Berufsgruppen- und Bereichsdenke“ hin zu einer offenen, prozessbezogenen Aufgabenverteilung.

Eine Vernetzung der Verantwortungsbereiche kann organisatorisch betrachtet nur „Vom Kopf“ her erfolgen. Eine Überbrückung der Berufsgruppengrenzen und $\mathrm{Zu}$ sammenführung der wesentlichen Expertisen im Stationskontext gelingt durch die Etablierung eines Managementtandems aus verantwortlichem Oberarzt und Stationsleitung. Ihnen obliegt die gemeinsame Prozess- und Managementverantwortung, sowie in enger Abstimmung die Personalverantwortung jeweils für die ärztlichen und pflegerischen Mitarbeiter sowie für das Unterstützungspersonal. Das Managementtandem ist von Beginn an eng in die Reorganisationsmaßnahmen einzubinden, denn nur so kann es wirksam die übergeordnete Prozessverantwortung auskleiden. Flankierende Schulungen oder Einzelcoachings, um die Führungskräfte auf ihre neue Rolle vorzubereiten, unterstützen den Veränderungsprozess.

Neustrukturierung der Aufgaben- und Kompetenzbereiche Nach der Festigung des Führungsteams erfolgt in einem nächsten Schritt die Neustrukturierung der Aufgaben- und Kompetenzbereiche entlang der Behandlungsprozesse auf den Stationen. Im Fokus der Gestaltung stehen die Kernprozesse Entlassung, Belegungssteuerung, Aufnahme und Visite sowie in einem Folgeschritt die Unterstützungsprozesse wie Arztbriefschreibung oder Übergaben. In berufsgruppenübergreifenden Projektgruppen werden die Prozesse auf den Prüfstand gestellt und optimiert. Neben der Neugestaltung der Ablaufstrukturen ist die Neuausrichtung der Aufgaben- und Kompetenzbereiche ein wesentliches Element der Organisationsveränderung auf Station. Die Einführung eines gestuften Personalkonzeptes hilft, ein vernetztes Stationsmanagement in den Vordergrund zu stellen. Die Ausgestaltung des Personalmixes muss den Anforderungen der Station gerecht werden. Diese sind je nach Fachgebiet oder auch Größe der Station unterschiedlich. Übergeordnete Zielsetzung ist die Refokussierung von Ärzten und Pflegemitarbeitern auf ihre Kerntätigkeiten, sowie die Definition von Aufgabenbereichen, die an Unterstützungspersonal delegiert werden können. Anhand der festgelegten Prozessschritte wird der Personaleinsatz neu bewertet und entsprechend umverteilt. Zudem gilt zu beachten, dass ein Zuviel an Unterstützungspersonal erheblichen Koordinationsaufwand mit sich bringt. Die Aufgabengebiete müssen klar abgegrenzt und aus der Perspektive der neu geschaffenen Stellen zu sinnvollen Einheiten zusammengefasst werden.

Abschließend werden die Einzelprozesse aufeinander synchronisiert (z. B. Neuaufnahmen erfolgen nach Beendigung des Entlasszeitfensters) und in einem berufsgruppenübergreifenden Tagesablauf dargestellt. Allein die visualisierte Darstellung über alle Berufsgruppen hinweg macht die Verzahnung der Prozesse und die gemeinsamen Verantwortungsbereiche deutlich (Abb.1). Darüber hinaus ist zu definieren, wer die Vorgesetztenfunktion für das neu eingeführte Unterstützungspersonal wahrnimmt.

Betriebswirtschaftliche Betrachtung Für die Festlegung eines optimierten Personalmixes gibt es kaum Anhaltszahlen. 


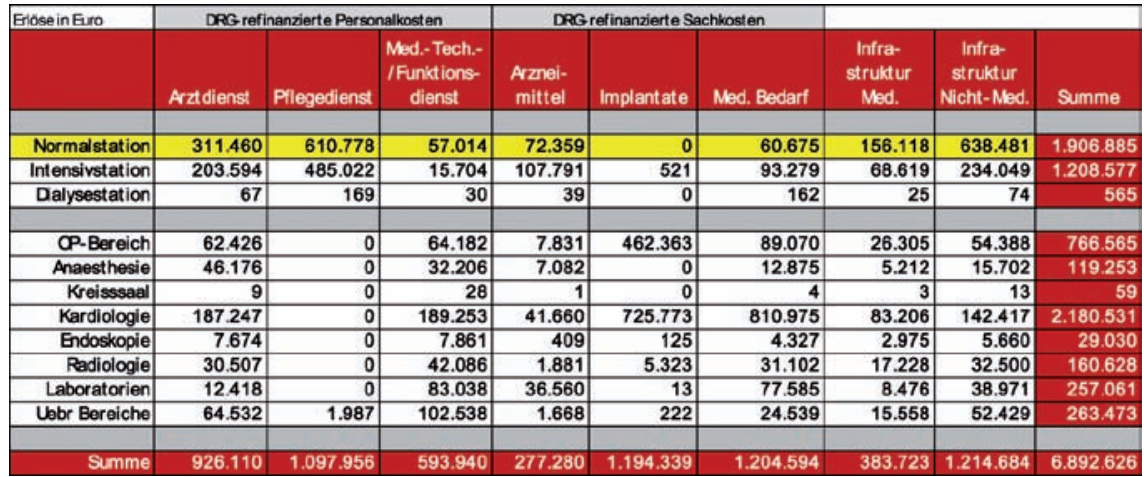

Tab. 1 Erlösmatrix nach DRG

\begin{tabular}{|r|r|r|r|r|}
\hline 2007 & Arzt dienst & Pflegedienst & $\begin{array}{c}\text { Med.- Tech.- } \\
\text { /Funktions- } \\
\text { dienst }\end{array}$ & Summe \\
\hline Normalstation & & & & \\
\hline Elöse in Euro & 288.816 & 597.955 & 64.154 & 950.924 \\
\hline Kosten in Euro & 303.851 & 534.533 & 0 & 838.384 \\
\hline & & & & \\
\hline Summe & -15.035 & 63.421 & 64.154 & 112.540 \\
\hline
\end{tabular}

Tab. 2 Personalbudget 2007

\begin{tabular}{|c|c|c|c|c|}
\hline & \multirow{2}{*}{2007} & Werktag & Wochenend & \multirow[b]{2}{*}{ W-Ges. } \\
\hline & & $\mathrm{K}^{*}$ & W & \\
\hline & Pflegedienst & 9,30 & 3,00 & 12,30 \\
\hline & Arzt dienst & 3,00 & 0,60 & 3,60 \\
\hline & Summe & & & 15,90 \\
\hline & \multirow{2}{*}{2008} & Werktag & Wochenende & \\
\hline & & $\mathrm{W}^{*}$ & WK & W-Ces. \\
\hline & Pflegedienst & 8,80 & 3,00 & 11,80 \\
\hline \multirow{5}{*}{$\begin{array}{l}\text { Gestuftes Pers.- } \\
\text { konzept }\end{array}$} & MDA & 0,50 & & 0,50 \\
\hline & Stationssekretärin & 1,00 & & 1,00 \\
\hline & Belegungsmanagement & 0,50 & & 0,50 \\
\hline & Arzt dienst & 2,70 & 0,60 & 3,30 \\
\hline & Summe & & & 16,60 \\
\hline
\end{tabular}

Tab. 3 Personalmix

\begin{tabular}{|r|r|r|r|r|}
\hline 2008 & Arzt dienst & Pflegedienst & $\begin{array}{c}\text { Med.-Tech.- } \\
\text { IFunktions- } \\
\text { dienst }\end{array}$ & Summe \\
\hline Normalstation & & & & \\
\hline Elöse in Euro & $\mathbf{3 1 1 . 4 6 0}$ & $\mathbf{6 1 0 . 7 7 8}$ & $\mathbf{5 7 . 0 1 4}$ & $\mathbf{9 7 9 . 2 5 2}$ \\
\hline Kosten in Euro & $\mathbf{2 8 9 . 6 7 1}$ & $\mathbf{5 7 0 . 1 6 9}$ & 19.805 & $\mathbf{8 7 9 . 6 4 5}$ \\
\hline & & & & \\
\hline Summe & 21.789 & 40.609 & 37.209 & 99.607 \\
\hline
\end{tabular}

Tab. 4 Personalbudget 2008

Einen betriebswirtschaftlichen Orientierungspunkt bilden die durch die DRG-Erlöse refinanzierten Personalbudgets, die einer Station zugewiesen werden kön- nisch-technischen Dienstes/ Funktionsdienstes ermittelt und in einer Erlösmatrix zusammengefasst werden (vgl. Tab. 1). Diese bildet sozusagen den Benchmark für die Verteilung des Personals, wie er durch die an der InEK-Kalkulation teilnehmenden Kliniken vorgegeben ist. $\mathrm{Zu}$ dem entspricht die Verteilung der durch die DRGs sichergestellten Finanzierung des Personals. Erlöse aus Wahlleistungen etc. müssen hier noch addiert werden. Um einen Vergleich der eigenen Strukturen vornehmen zu können, müssen die Kosten nach der gleichen Systematik auf die einzelnen Module verteilt werden. Für das genaue Vorgehen zur Ermittlung der Kostenmodule sei auf das Kalkulationshandbuch der InEK verwiesen. Im Unterschied zu den dort beschriebenen Umlageschritten muss für die Stationsauswertung eine Zuweisung zu der jeweiligen Abteilung/Station und nicht auf den Einzelfall erfolgen.

Bei der Gegenüberstellung von Erlösen und Kosten ergeben sich Anhaltspunkte für die Einführung gestufter Personalkonzepte. Dies verdeutlicht das folgende Beispiel.

Vor Durchführung der Organisationsveränderung auf Station zeigte eine Gegenüberstellung des DRG-refinanzierten Personalbudgets mit den tatsächlichen Personalkosten folgendes Bild (Tab. 2).

Bei der Prozessreorganisation wurde ein besonderes Augenmerk auf Tätigkeiten gelegt, die den Arztdienst entlasten, um die dort auftretende Unterdeckung der Personalkosten auszugleichen. Zur direkten Unterstützung wurde eine Medizinische Dokumentationsassistentin mit 0,5 Vollkräften eingesetzt, die neben der Kodierung auch eine wichtige Rolle beim Verweildauermanagement einnimmt. Der Arztdienst wurde um 0,3 Vollkräfte reduziert, die freiwerdenden Ressourcen für die Verstärkung in der Ambulanz, einem Engpassfaktor der Abteilung, eingesetzt.

In einem weiteren Schritt wurde ein Stationssekretariat auf der Station etabliert. Das Stationssekretariat übernimmt primär administrative Tätigkeiten. Die Unterstützungsleistung bezieht sich auf die Kernprozesse der Station und erfolgt berufsgruppenübergreifend. Das Stationssekretariat entlastet somit Ärztlichen Dienst und Pflegedienst gleichermaßen. Zum Aufgabenportfolio zählt z. B. Unterstützung bei der Arztbriefschreibung (Übernahme von Befunden etc.), admi- 
nistrative Aufnahme von Patienten, Telefonmanagement auf Station, Vorbereitung sämtlicher Patientenunterlagen oder je nach Berufsausbildung (z. B. Arzthelferin) auch Unterstützung bei der Blutabnahme. Darüber hinaus wurde eine halbe Stelle aus dem bisherigen Pflegepersonal für das Belegungsmanagement auf der Station zugewiesen und damit ein zentraler Ansprechpartner für alle einweisenden Stellen geschaffen.

Ein Vorher-/ Nachher-Abgleich der Vollkräfte auf Station gibt das in Tabelle 3 gezeigte Bild. Die erneute Auswertung des Personalbudgets nach einem Jahr wird in Tabelle 4 gezeigte.

Durch die Einführung des gestuften Personalkonzeptes konnte eine verursachungsgerechtere Abbildung der Personalkosten erreicht werden. Die Personalbudgets der einzelnen Berufsgruppen liegen innerhalb des durch die DRGs vorgegebenen Benchmarks und weisen einen Überschuss aus. Dabei erfolgte die Personalmaßnahme als solches kostenneutral. Im Vergleich zur Ausgangssituation sind die Personalkosten um ca.
28000 Euro gestiegen. Im Gegenzug wurden die Vollkräfte um ca. 0,7 VK aufgestockt. Zudem fand eine Lohnsteigerung in Höhe von ca. 3\% statt.

In die betriebswirtschaftlichen Erwägungen müssen die Auswirkungen der Aufgabenverteilung und die damit einhergehenden strukturellen Veränderungen unter weiteren Gesichtspunkten bewertet werden. Insgesamt konnte in der Abteilung durch die eingeführten Veränderungen eine Erlössteigerung realisiert werden, die allein auf Station ca. 41200 Euro im Bereich der erlösfinanzierten Personalbudgets ausmachte. Die Erlössteigerungen resultierten unter anderem aus der optimierten Belegungssteuerung sowie aus durch die Prozessreorganisation erreichten Verweildauereffekten.

Bei der betriebswirtschaftlichen Bewertung der Einführung von gestuften Personalkonzepten und der Umverteilung von Aufgaben und Kompetenzen bieten die DRG-erlösfinanzierten Personalbudgets eine Richtgröße zur Überprüfung. Gleichzeitig müssen Erlössteigerungen oder
Kennzahlen wie Verweildauereffekte, Auswirkungen auf Ausfallquoten der Mitarbeiter oder Prozesskennzahlen in die Erfolgsrechnung einbezogen werden. Die Definition der zugrundeliegenden Kennzahlen bestimmt sich immer auch durch die Zielsetzung der durchgeführten Maßnahmen und erweitert die reine Kostenund Erlösbetrachtung.

\section{Korrespondenz}

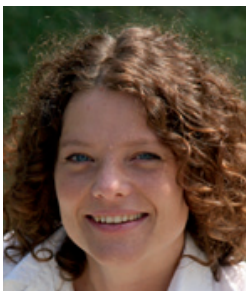

Nicole Krüttgen Ruhl Consulting AG

Harrlachweg 1

68163 Mannheim

Telefon 0621 328850-0

E-Mail: info@ruhl-consulting.de

www.ruhl-consulting.de

Blog: http://blog.ruhl-consulting.de

News: http://newsletter.ruhl-consulting.de 